ORIGINAL ARTICLE

\title{
Cytokeratin phenotyping does not help in distinguishing oesophageal adenocarcinoma from cancer of the gastric cardia
}

\author{
M G F van Lier, F J Bomhof, I Leendertse, M Flens, A T Balk, R J L F Loffeld
}

J Clin Pathol 2005;58:722-724. doi: 10.1136/icp.2004.024265

See end of article for authors' affiliations .....................

Correspondence to: DrR J L F Loffeld Department of Internal Medicine, De Heel Zaans Medisch Centrum, PO Box 210, 1500 EE Zaandam, The Netherlands;

r.loffeld@chello.n

Accepted for publication 15 January 2005
Background: It is sometimes difficult to distinguish between cardia cancer and oesophageal cancer. Aims: To evaluate whether cytokeratin (CK) expression of the tumour can be of value in differentiating between the two tumour types.

Methods: Consecutive patients with a malignant tumour in the oesophagus or stomach were recruited. Biopsy specimens were taken for routine haematoxylin and eosin staining. One tissue block with representative tissue was selected for immunohistochemical staining (CK7 and CK20).

Results: Endoscopically located adenocarcinoma of the oesophagus was present in 84 patients (64 men, 20 women; mean age, 68 years; range, 44-91). Cancer located primarily in the gastric cardia was present in 63 patients (42 men, 21 women; mean age, 68 years; range, 42-88). The histological diagnosis was metastasis from a primary tumour outside the oesophagus or stomach in 19 patients. The patients were divided into three groups for the immunohistochemical analysis. Patients in group A had definite oesophageal cancer, group B patients had a definite carcinoma located in the gastric cardia, and group $C$ patients had an obstructing tumour distal in the oesophagus at the level of the diaphragm, which could not be passed with the endoscope. Paraffin wax embedded material was available from 122 patients for immunostaining and CK analysis. There was no significant difference in expression or distribution of CK7 or CK2O in the three groups of patients.

Conclusion: CK phenotyping cannot distinguish between cancer arising from a Barrett's oesophagus and carcinoma originating in the gastric cardia.
A denocarcinoma in the distal part of the oesophagus and the gastric cardia is increasing in incidence. ${ }^{12}$ Barrett's oesophagus is the replacement of the squamous mucosa by metaplastic columnar lined glandular epithelium. Intestinal metaplasia in the oesophagus is a definite risk factor for developing cancer. It is considered a premalignant condition. ${ }^{3-5}$ However, in daily practice, all patients in whom an adenocarcinoma in the distal oesophagus is diagnosed have not previously been diagnosed with Barrett's metaplasia.

"Clinically, and during endoscopy, it is sometimes difficult to distinguish between an adenocarcinoma in the oesophagus and a malignant tumour originating in the gastric cardia $^{\prime \prime}$

The best available treatment for cancer in the oesophagus and proximal stomach is surgical resection. Clinically, and during endoscopy, it is sometimes difficult to distinguish between an adenocarcinoma in the oesophagus and a malignant tumour originating in the gastric cardia.

The cytokeratins (CKs) are a family of cytoplasmic structural proteins that have been described in normal epithelium and in many tumours. CK20 is a marker of intestinal differentiation, and is expressed at the surface and in the crypt epithelium of the intestine. CK7 is claimed to be present in tissue originating from intestinal metaplasia in the oesophagus.

We studied consecutive patients diagnosed with oesophageal adenocarcinoma and cancer located in the gastric cardia to evaluate whether CK expression can be of value in differentiating between the two tumour types.

\section{MATERIAL AND METHODS}

\section{Patients}

All consecutive patients with a malignant tumour in the oesophagus or stomach diagnosed from 1992 to 2003 in the Zaans Medical Centre, the community hospital of the Zaanstreek region, the Netherlands, were included. All patients underwent upper gastrointestinal endoscopy. During the procedure, biopsy specimens were taken for routine haematoxylin and eosin staining to confirm the macroscopic diagnosis. The anatomical localisation of the tumour was noted. A Barrett's oesophagus was judged to be present if the typical columnar lined epithelium was seen in the tubular oesophagus. A hiatus hernia was defined as a distance of more than $2 \mathrm{~cm}$ between the oesophago-gastric junction and the diaphragm.

\section{Immonuhistochemistry}

All paraffin wax embedded biopsy specimens were retrieved. One tissue block with representative tissue was selected for immunohistochemical staining. The formalin fixed, paraffin wax embedded sections were dewaxed. To enhance immunostaining, these sections were subjected to enzyme digestion for epitope retrieval using protease 1 (Ventana Medical Systems, Tucson, Arizona, USA). Endogenous peroxidase and non-specific binding were blocked before addition of the primary antibody.

Sections were then immunostained using the Ventana ES automated immunohistochemistry system, using antibodies to CK7 and CK20. Monoclonal mouse (IgG) antihuman CK20 clone Ks20.8 (Dako, Glostrup, Denmark) and monoclonal

Abbreviations: CK, cytokeratin 


\begin{tabular}{|c|c|c|c|}
\hline & \multicolumn{3}{|l|}{ Group } \\
\hline & A & B & C \\
\hline $\begin{array}{l}\text { Number of patients } \\
\text { CK7 }\end{array}$ & 28 & 47 & 47 \\
\hline+ & $25(89)$ & $41(87)$ & $43(91)$ \\
\hline Insufficient material ${ }^{*}$ & $\begin{array}{l}1 \\
2\end{array}$ & $\begin{array}{l}5 \\
1\end{array}$ & $\begin{array}{l}4 \\
-\end{array}$ \\
\hline Diffuse & 23 (92) & $34(83)$ & $41(95)$ \\
\hline $\begin{array}{l}\text { Focal } \\
\text { CK20 }\end{array}$ & $2(8)$ & $7(17)$ & $2(5)$ \\
\hline+ & $19(68)$ & $29(62)$ & $34(72)$ \\
\hline- & 7 & 17 & 13 \\
\hline Insufficient material* & 2 & 1 & - \\
\hline Diffuse & $9(47)$ & $11(38)$ & $11(32)$ \\
\hline Focal & $10(53)$ & $18(62)$ & $23(68)$ \\
\hline
\end{tabular}

mouse (IgG) antihuman CK7 clone OV-TL 12/30 (Biogenex, San Ramon, California, USA) were both diluted $1 / 200$.

The appropriate biotinylated secondary antibodies (affinity purified goat antimouse IgG and IgM and goat antirabbit IgG in phosphate buffer with a preservative) were detected using an iVIEW DAB (3,3-diaminobenzidine tetrahydrochloride) conjugate. The complex was visualised with a hydrogen peroxidase substrate and a DAB chromogen plus a copper enhancer. This produces a dark brown precipitate detectable by light microscopy. The specimens were counterstained using Mayer's haematoxylin (Klinipath, Duiven, the Netherlands) and cover slipped.

\section{Evaluation of CK immunostaining}

All immunohistochemical stains were evaluated, without specific knowledge of the anatomical localisation of the tumour, by two observers. The distribution and intensity of cytoplasmic CK7 and CK20 staining in neoplastic cells were noted. Staining intensity was determined at $\times 250$ and $\times 400$ magnification in a semiquantitative fashion as absent $(-)$, low $( \pm)$, intermediate $(+)$, or high $(++)$. The distribution was noted as focal or diffuse.

\section{Statistics}

Statistical analysis was carried out by means of the $\chi^{2}$ test for contingency tables. A p value below 0.05 was considered significant.

\section{RESULTS}

In the defined period of time, 370 consecutive patients were seen with cancer of the oesophagus or stomach. Distally located stomach cancer was diagnosed in 175 patients (107 men, 68 women; mean age, 73 years; range, 27-97). These patients were excluded from further analysis. Endoscopically located adenocarcinoma of the oesophagus was present in 84 patients (64 men, 20 women; mean age, 68 years; range, 4491). Thirty eight patients had a squamous cell carcinoma in the oesophagus (27 men, 11 women; mean age, 65 years; range, 40-87). Cancer located primarily in the gastric cardia was present in 63 patients ( 42 men, 21 women; mean age, 68 years; range, 42-88). The histological diagnosis was metastasis from a primary tumour outside the oesophagus or stomach in 19 patients.

An obstructing tumour in the distal oesophagus that could not be passed with the endoscope was scored endoscopically as an oesophageal cancer. For this reason, the patients were divided into three groups for the immunohistochemical analysis. Group A patients had definite oesophageal cancer
Table 2 The semiquantitative expression of cytokeratins (CKs) in the three groups of patients

\begin{tabular}{llll}
\hline \multicolumn{4}{c}{ Group } \\
\cline { 2 - 4 } & A & B & C \\
\hline CK7 & & $3(7)$ & $1(2)$ \\
Low & - & $5(12)$ & $11(26)$ \\
Intermediate & $3(12)$ & $33(81)$ & $31(72)$ \\
High & $22(88)$ & $3(10)$ & $3(9)$ \\
CK20 & $1(5)$ & $22(76)$ & $21(62)$ \\
Low & $13(68)$ & $4(14)$ & $10(29)$ \\
Intermediate & $5(27)$ & \\
High &
\end{tabular}

because of the macroscopic presence of Barrett's metaplasia and/or the presence of a hiatus hernia. Group B consisted of patients with a definite carcinoma located in the gastric cardia, which was identified by means of a U turn during endoscopy, and finally, group C patients had an obstructing tumour distal in the oesophagus at the level of the diaphragm, which could not be passed with the endoscope.

Paraffin wax embedded material was available from 122 patients for immunostaining and CK analysis, from 28 patients in group A, 47 in group B, and 47 in group C.

Tables 1 and 2 show the CK expression phenotype and intensity of staining in the three groups of patients. There was no significant difference between the groups for either of the CKs studied. In addition, the combined CK7/CK20 phenotype (CK7+/CK20+, CK7-/CK20+, CK7+/CK20-, and CK7-/CK20-) was not helpful in distinguishing between definite oesophageal adenocarcinoma and cardia cancer.

\section{DISCUSSION}

There are several studies in the literature on CK expression in Barrett's epithelium and the gastric cardia. Intestinal metaplasia in Barrett's oesophagus and intestinal metaplasia of the gastric cardia can be distinguished on the basis of CK7 and CK20 expression. ${ }^{6-8}$ The largest study ${ }^{7}$ showed that a CK7+/CK20 - phenotype had a positive predictive value of $87 \%$ for the diagnosis of adenocarcinoma of the distal oesophagus. About $90 \%$ of Barrett's cancers were positive for CK7 but less than $18 \%$ expressed CK20. ${ }^{7}$ The same phenotype was found in only $24 \%$ of cancers located in the proximal stomach. Others confirmed these results. ${ }^{9-11}$ However, other studies have produced contradictory results, and have shown that CK7 but and CK20 immunoreactivity did not differentiate between Barrett's adenocarcinoma and gastric cancer located at the cardia. ${ }^{12-15}$ Our present study is in line with these results. In one study, the CK phenotype was used to try to differentiate short segment Barrett's intestinal metaplasia from cardia intestinal metaplasia, but the investigators could not differentiate between the two conditions. ${ }^{14}$ However, specific biopsy specimens from a short segment Barrett's oesophagus are almost impossible to obtain, because the area of interest moves constantly during biopsy taking. Every experienced endoscopist will confirm this. Hence sampling errors are bound to occur.

What can be the reason for the discrepancies in the literature? Bias and possible confounding factors hamper these studies. Most studies reported on small numbers and did not use strict topographical endoscopic criteria for localisation of the biopsy sites. Both biopsy specimens and resection specimens have been studied, as have biopsies from the tumour and from normal epithelium adjacent to the carcinoma, and patients with cancer and those with intestinal metaplasia. In one study, ${ }^{9}$ biopsy specimens and surgical resections were studied. However, patients with an 


\section{Take home messages}

- Because it can sometimes be difficult to distinguish between oesophageal cancer and gastric cardia carcinoma we investigated the expression of cytokeratin 7 (CK7) and CK20 in these two entities

- CK phenotyping was not able to distinguish between cancer arising from a Barrett's oesophagus and carcinoma originating in the gastric cardia

inoperable tumour were not included. Our present study is a clinical study. Patients were retrieved from endoscopy files. The endoscopist was the only person who knew the in vivo anatomical localisation of the tumour from which the biopsy specimens were taken. Endoscopy was used as gold standard with regard to tumour localisation. In our opinion, this is the only correct way to carry out such studies because most patients are inoperable at the time of diagnosis. Radiological studies are not the proper way to identify the exact localisation of the tumour. This is especially true for large tumours. Endoscopy also has its problems, especially if the tumour cannot be passed with the endoscope. However, if an endoscope can be introduced into the stomach a proper identification of the primary localisation of the cancer will be possible. For this reason, in our current study three groups of patients were identified.

"Specific biopsy specimens from a short segment Barrett's oesophagus are almost impossible to obtain, because the area of interest moves constantly during biopsy taking"

The presence of a hiatus hernia is very helpful in the anatomical localisation of the tumour. In our experience, $82 \%$ of patients presenting with a Barrett's oesophagus also have a hiatus hernia. ${ }^{16}{ }^{17}$ Given this figure, and the knowledge that adenocarcinoma in the oesophagus develops from Barrett's oesophagus, it is conceivable that a large number of patients with adenocarcinoma in the distal part of the oesophagus will have a hiatus hernia. However, this anatomical abnormality often cannot be visualised because of a large tumour mass, or even an obstructing tumour that makes further introduction of the endoscope impossible. Hence, group C consisted of patients with true oesophageal cancer and obstructing cardia cancer.

In our present study, the CK phenotype of cancers in the oesophagus and gastric cardia was studied. It is important to use a well known and accepted method of immunostaining. Most studies have used the monoclonal antibody Ks20.8 to detect CK20, as we did. CK20 has been reported to be positive predominantly in the superficial parts of Barrett's mucosa, ${ }^{18}$ although this pattern could not be confirmed in our present study. CK20 expression was seen throughout the tumour. In Barrett's mucosa, CK7 is diffusely positive in superficial and deep glands, ${ }^{19}$ whereas CK20 is positive in the surface epithelium and superficial glands. Patchy CK7 immunostaining is seen in the gastric cardia, with variable involvement of deep glands, and CK20 shows patchy immunostaining of superficial and deep glands. ${ }^{19}$ Our present study shows that at least in cancers originating from Barrett's mucosa and the gastric cardia this pattern is not seen.
The definition of subgroups according to the site of development may prove to be valuable for clinical diagnosis and treatment. Using the above mentioned criteria as the gold standard it was not possible to detect differences in CK phenotype between oesophageal adenocarcinoma and cardia carcinoma.

In conclusion, the CK phenotype cannot distinguish between cancer arising form a Barrett's oesophagus or carcinoma originating in the gastric cardia.

\section{Authors' affiliations}

M G F van Lier, R J L F Loffeld, Department of Internal Medicine, De Heel Zaans Medisch Centrum, 1500 EE Zaandam, The Netherlands

F J Bomhof, I Leendertse, M Flens, A T Balk, Department of Pathology, De Heel Zaans Medisch Centrum

\section{REFERENCES}

1 Pera M, Cameron AJ, Trastek VF, et al. Increasing incidence of adenocarcinoma of the esophagus and esophagogastric junction. Gastroenterology 1993; 104:510-13.

2 Hansson LE, Sparen P, Nyren O. Increasing incidence of both major histological types of esophageal carcinomas among men in Sweden. Int J Cancer 1993;54:402-7.

3 Blot W, Devesa SS, Fraumeni JF. Continuing climb in rates of esophageal adenocarcinoma: an update. JAMA 1993;270:1320.

4 Prach AT, MacDonal TA, Hopwood DA, et al. Increasing incidence of Barrett's oesophagus: education, enthusiasm, or epidemiology? Lancet 1997;350:933.

5 Cameron AJ. Epidemiology of columnar-lined esophagus and adenocarcinoma. Gastroenterol Clin North Am 1997;26:487-93.

6 Sarbia M, Donner A, Franke C, et al. Distinction between intestinal metaplasia in the cardia and in Barrett's esophagus: the role of histology and immunohistochemistry. Hum Pathol 2004;35:371-6.

7 Shen B, Ormsby AH, Shen C, et al. Cytokeratin expression patterns in noncardia, intestinal metaplasia-associated gastric adenocarcinoma: implication for the evaluation of intestinal metaplasia and tumors at the esophagogastric junction. Cancer 2002;94:820-31.

8 Ormsby AH, Goldblum JR, Rice TW, et al. Cytokeratin subsets can reliably distinguish Barrett's esophagus from intestinal metaplasia of the stomach. Hum Pathol 1999:30:288-94.

9 Taniere P, Borghi-Scoazec G, Saurin JC, et al. Cytokeratin expression in adenocarcinomas of the esophagogastric junction: a comparative study of adenocarcinomas of the distal esophagus and the proximal stomach. Am J Surg Pathol 2002;26:1213-21.

10 Ormsby AH, Goldblum JR, Rice TW, et al. The utility of cytokeratin subsets in distinguishing Barrett's related oesophageal adenocarcinoma from gastric adenocarcinoma. Histopathology 2001;38:307-11.

11 Jovanovic I, Tzardi M, Mouzas IA, et al. Changing pattern of cytokeratin 7 and 20 expression from normal epithelium to intestinal metaplasia of the gastric mucosa and the gastroesophageal junction. Histol Histopathol 2002; 17:445-54

12 Flucke U, Steinborn E, Dries $\mathrm{V}$, et al. Immunoreactivity of cytokeratins (CK7, CK20) and mucin peptide core antigens ( $\mathrm{MUC1}, \mathrm{MUC2}, \mathrm{MUC5AC}$ ) in adenocarcinomas, normal and metaplastic tissue of the distal oesophagus, oesophago-gastric junction and proximal stomach. Histopathology 2003;43:127-34

13 DeMeester SR, Wickramasinghe KS, Lord RV, et al. Cytokeratin and DAS-1 immunostaining reveal similarities among cardiac mucosa, CIM, and Barrett's esophagus. Am J Gastroenterol 2002;97:2514-23.

14 Mohammed IA, Streutker CJ, Riddell RH. Utilization of cytokeratins 7 and 20 does not differentiate between Barrett's esophagus and gastric cardiac intestinal metaplasia. Mod Pathol 2002;15:61 1-16.

15 Gulmann C, Counihan I, Grace A, et al. Cytokeratin 7/20 and mucin expression patterns in oesophageal, cardia and distal gastric adenocarcinomas. Histopathology 2003;43:453-61.

16 Werdmuller B, van der Putten ABMM, Loffeld RJLF. The presentation of gastroesophageal reflux disease: a prospective clinical and endoscopic study. Dis Esophagus 1996;9:285-9.

17 Loffeld RJLF, ABMM van der Putten. The yield of upper gastrointestinal endoscopy: a study of a ten-year period in the "Zaanstreek". Neth J Med 2003;61:18-22.

18 Mandys V, Lukas K, Revoltella R. Different patterns of cytokeratin expression in Barrett's esophagus - what is beyond? Pathol Res Pract 2003; 199:581-7.

19 Kurtkaya-Yapicier O, Gencosmanoglu R, Avsar E, et al. The utility of cytokeratins 7 and 20 (CK7/20) immunohistochemistry in the distinction of short-segment Barrett esophagus from gastric intestinal metaplasia: is it reliable? BMC Clin Pathol 2003;2:3-5. 